\title{
Particle flow and reaction plane reconstruction in the CBM experiment
}

\author{
V. Mikhaylov ${ }^{* \dagger}$ \\ Nuclear Physics Institute, Czech Academy of Sciences, Řež, Czech Republic \\ Czech Technical University in Prague, Faculty of Nuclear Sciences and Physical Engineering, \\ Prague, Czech Republic \\ National Research Tomsk Polytechnic University, Department of Electronics and Automation of \\ Nuclear Plants, Tomsk, Russia (on leave from)
}

\section{A. Kugler, V. Kushpil, P. Tlustý}

Nuclear Physics Institute, Czech Academy of Sciences, Řež, Czech Republic

\section{S. Seddiki}

GSI Helmholtzzentrum für Schwerionenforschung GmbH, Darmstadt, Germany

\section{Selyuzhenkov}

GSI Helmholtzzentrum für Schwerionenforschung GmbH, Darmstadt, Germany

National Research Nuclear University MEPhI, Moscow, Russia

\begin{abstract}
Particle flow and reaction plane reconstruction performance using the Projectile Spectator Detector (PSD) in the CBM experiment at the future FAIR facility will be presented. The PSD is a compensating lead-scintillator calorimeter designed to measure the energy distribution of the forward going projectile nucleons and nuclei fragments (spectators) produced close to the beam rapidity. The main purpose of the PSD is to provide experimental estimates of heavy-ion collision centrality and reaction plane orientation. Directed and elliptic proton flow are simulated for $\mathrm{Au}+\mathrm{Au}$ collisions using five heavy-ion collision event generators: $\mathrm{QQMD}, \mathrm{UrQMD}, \mathrm{DCM}-\mathrm{QGSM}$, LA-QGSM and HSD. Reaction plane reconstruction performance was investigated for produced particles transported with the GEANT Monte-Carlo through realistic CBM detector geometry. Simulations are performed for the range of beam energies between 1 and $30 \mathrm{AGeV}$, which covers the expected beam energy range of the SIS100 and the SIS300 accelerator rings at FAIR. Results are compared with the experimental data from FOPI, HADES, AGS E877, E895 and STAR.
\end{abstract}

The European Physical Society Conference on High Energy Physics

22-29 July 2015

Vienna, Austria

\footnotetext{
${ }^{*}$ Speaker.

†email: mikhaylov@ujf.cas.cz
} 


\section{Introduction}

The collective motion of the final-state hadrons resulting from heavy-ion reactions contains important information on the collision dynamics. The isotropic, radial flow allows to characterize the collision system at kinetic freeze-out, i.e. when elastic collisions of the produced particles cease. Anisotropic flow results from the conversion of anisotropies in the density distribution into pressure gradients, and thus gives access to the equation of state of dense nuclear matter. At AGS, the experiment E895 measured the proton elliptic flow and reported a transition from outof-plane to in-plane emission at about $6 \mathrm{AGeV}$ [1]. The data indicate an evolution from a stiff equation-of-state below $2 \mathrm{AGeV}$ to a softer one at higher beam energies. Such softening of the EOS is suggestive of a phase transition to a deconfined state. Flow measurements by the FOPI Collaboration at SIS18, however, seem to exclude a stiff equation of state [2], a conclusion which is in line with earlier results on kaon production by KaoS and FOPI Collaborations [3].

In order to understand the degree of thermalisation, the features of equation of state, and the in-medium properties of strange particles in collisions of different systems at beam energies in the SIS100 energy range, a multi-differential flow measurements are needed for a number of hadron species, in particular for strange (anti-)baryons [4]. Such experimental program requires a largeacceptance hadron spectrometer, good particle identification through time-of-flight measurements and decay topology, the determination of the reaction plane and collision centrality with good accuracy, and high statistics for systematic studies in terms of system size and beam energy.

One of the facilities which will allow the study collective flow in the beam energy range $E_{b}=2-35 \mathrm{AGeV}$ is the future Facility for Antiproton and Ion Research (FAIR) in Darmstadt, Germany. It is designed to provide a high-intensity heavy-ion beams with SIS100/SIS300 accelerator rings [5]. FAIR will allow for unique research opportunities in the fields of nuclear, hadron, atomic and plasma physics. The Compressed Baryonic Matter (CBM) experiment at FAIR is designed to work in a high luminosity environment and will allow for a precision systematic measurement of production yields, phase-space distributions, correlations, and fluctuation observables for various particle species produced in nuclear collisions measurements [6]. Construction of FAIR and operation of the CBM experiment will start with primary beams delivered by the SIS100 synchrotron which is capable of accelerating gold nuclei up to the beam energy of $E_{b} \sim 11 \mathrm{AGeV}$.

The study of collective flow requires the measurement of an event plane which can be done with the Projectile Spectator Detector (PSD) of the CBM experiment [7]. The PSD is a compensating lead-scintillator calorimeter designed to measure the energy distribution of the projectile nuclei fragments (spectators) and forward going particles produced close to the beam rapidity. The main design requirements of the PSD are forward rapidity coverage and sufficient energy resolution to allow for precise collision centrality determination and consequently of the number of participating nucleons and granularity in the plane transverse to the beam direction which is needed for the collision symmetry plane reconstruction. The proposed 44 module design of the PSD covers large transverse area around the beam spot position such that most of the projectile spectator fragments deposit their energy in the PSD (Fig. 1).

This article reports the CBM performance for the measurement of directed and elliptic flow of protons in $\mathrm{Au}+\mathrm{Au}$ collisions at FAIR energies using GEANT Monte-Carlo simulation of the CBM detector response and physics input from various event generators. Simulations are performed for 

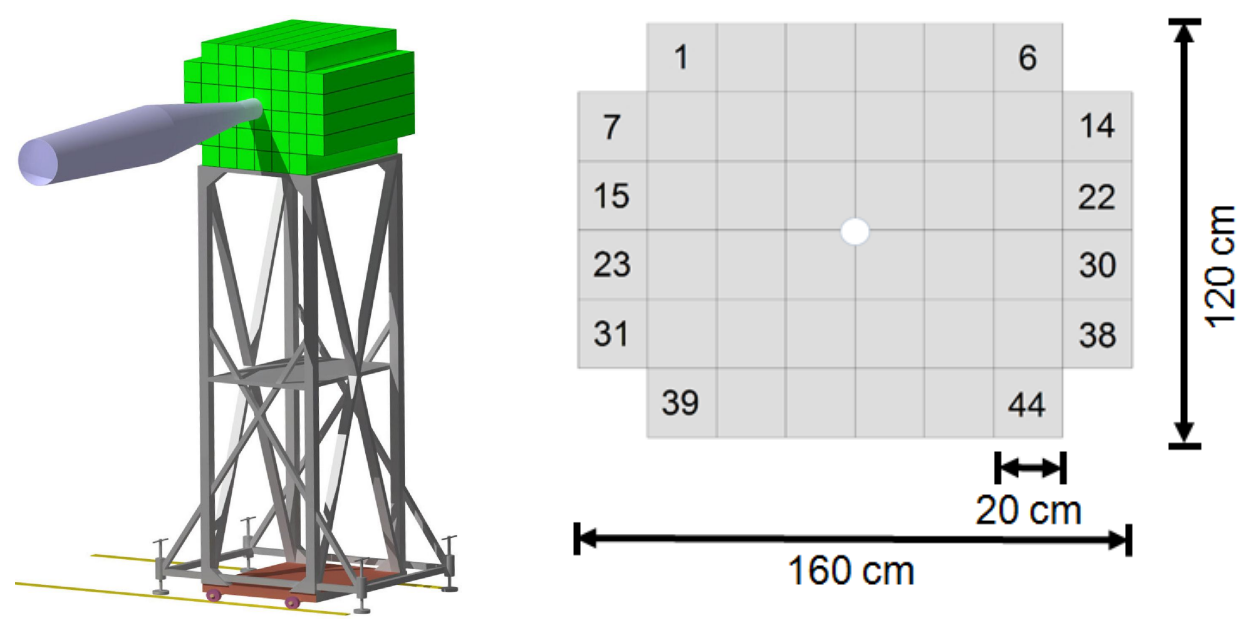

Figure 1: Layout of the Projectile Spectator Detector for the CBM experiment.

$E_{b}$ from 1 to $30 \mathrm{AGeV}$. The collision reaction plane is estimated from the simulated PSD response. Results of the directed and elliptic flow simulations as well as the reaction plane reconstruction performance are compared with the data from FOPI and HADES at GSI, E877 and E895 at AGS, and STAR at RHIC experiments.

\section{Collective flow of protons: models comparison with experiment data}

Directed and elliptic flow in $\mathrm{Au}+\mathrm{Au}$ collisions are compared for five different heavy-ion collision event generators, namely iQMD [8], UrQMD [9], HSD [11], DCM-QGSM and LAQGSM [10]. Simulations are performed for semi-central Au+Au collisions in the projectile beam energy range $E_{b}=1-30 \mathrm{AGeV}$, which covers the range from SIS18 at GSI up to SIS100/SIS300 at FAIR.
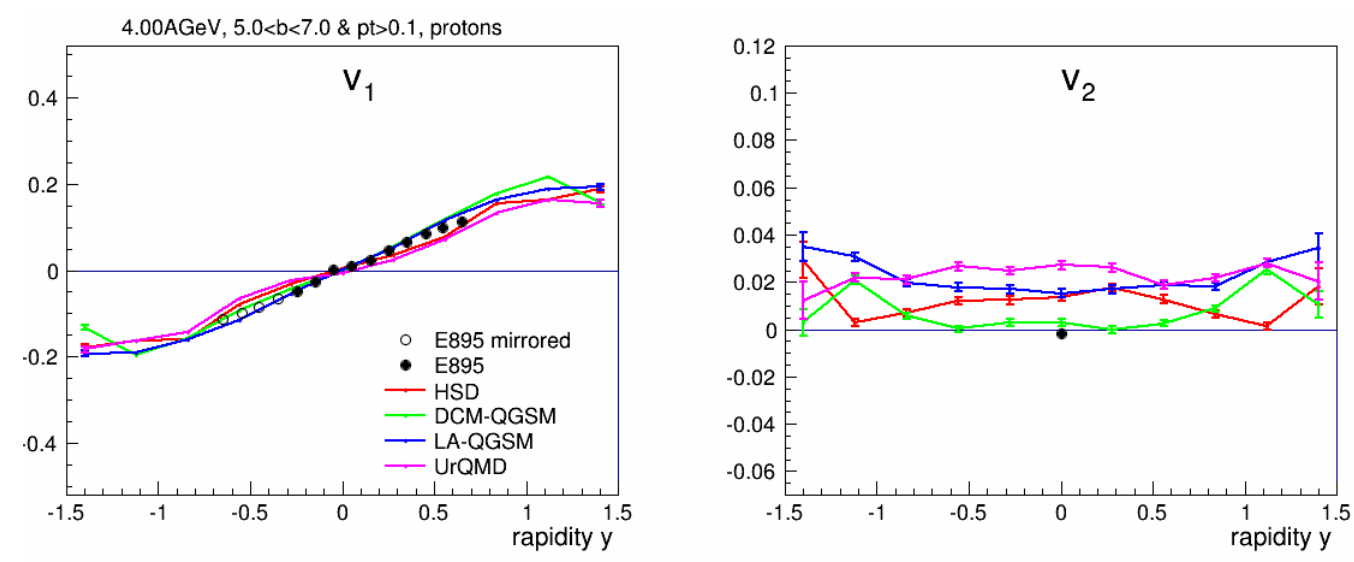

Figure 2: Directed (left) and elliptic (right) proton flow vs rapidity at $4 \mathrm{AGeV}$. 
An example of the collective flow of protons as function of rapidity at the SIS100 energies $\left(E_{b}=4\right.$ and $8 \mathrm{AGeV}$ ) is shown in Fig. 2 and Fig. 3. Collisions were simulated for impact parameter range $b=5-7 \mathrm{fm}$. Particles were selected with transverse momentum $p_{\mathrm{T}} \geq 0.1$ for $E_{b}=4 \mathrm{AGeV}$ and $p_{\mathrm{T}} \geq 0.4$ for $E_{b}=8 \mathrm{AGeV}$.
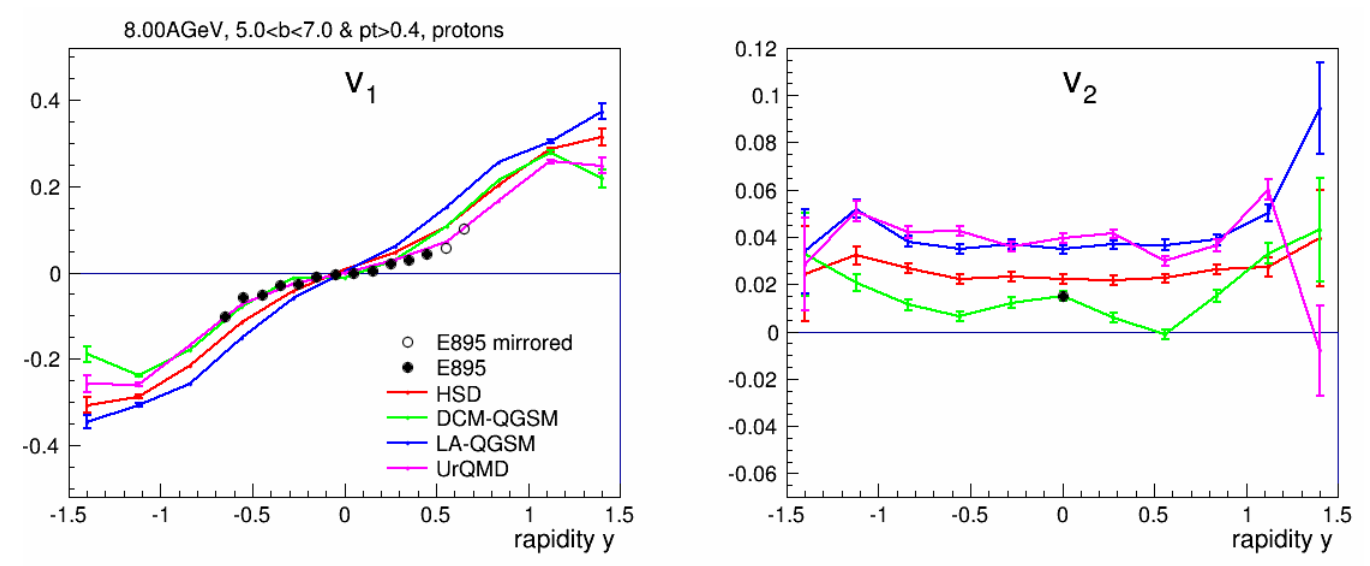

Figure 3: Directed (left) and elliptic (right) proton flow vs rapidity at $4 \mathrm{AGeV}$.

The slope of proton directed flow $\left(d v_{1} / d y\right)$ and elliptic flow $\left(v_{2}\right)$ at midrapidity as function of beam energy $E_{b}$ simulated with different generators is compared in Fig. 4 with FOPI [2], HADES [12], E895 [13] and STAR [14] experimental data. The impact parameter range is chosen
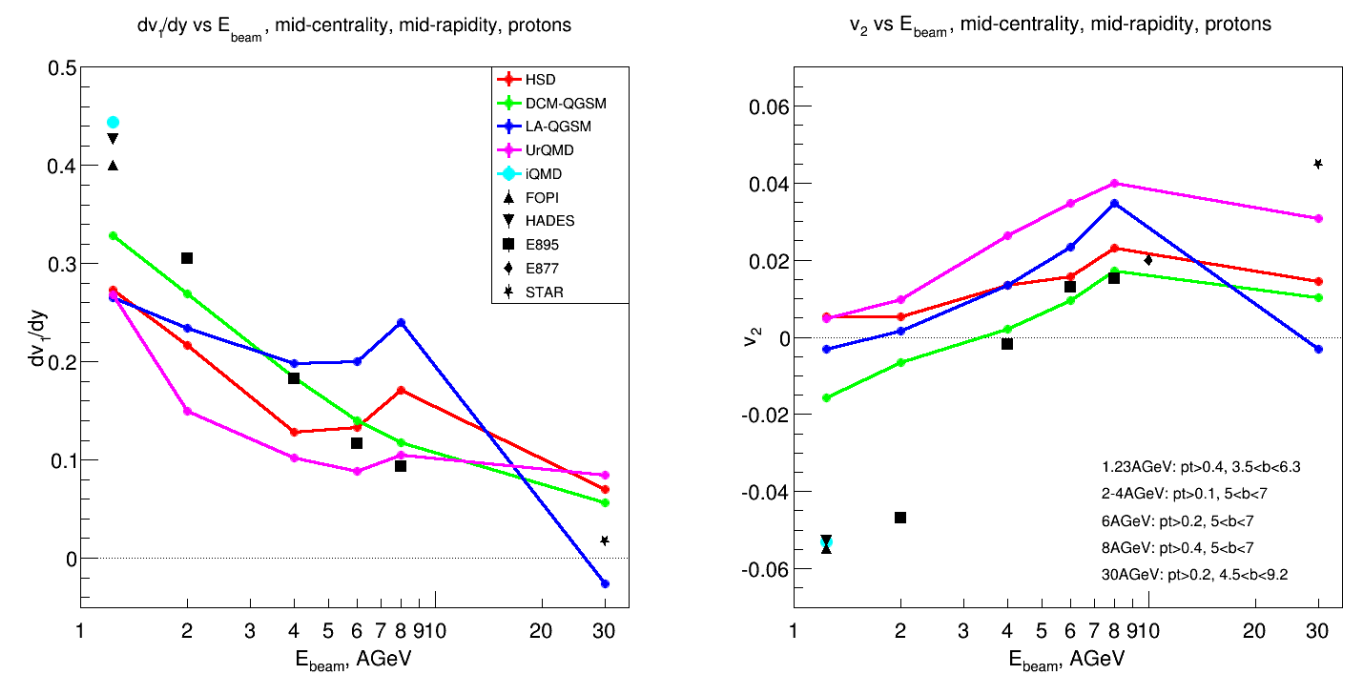

Figure 4: Directed (left) and elliptic (right) proton flow at midrapidity vs beam energy.

to be $b=3.3-6.0 \mathrm{fm}$ for $E_{b}=1.23 \mathrm{AGeV}, b=5-7 \mathrm{fm}$ for $E_{b}=2-8 \mathrm{AGeV}$, and $b=4.5-9.2 \mathrm{fm}$ for $E_{b}=30 \mathrm{AGeV}$. The lower $p_{\mathrm{T}}$ cut is $0.3 \mathrm{GeV} / \mathrm{c}$ for $E_{b}=1.23 \mathrm{AGeV}, 0.1 \mathrm{GeV} / \mathrm{c}$ for $E_{b}=2$, $4 \mathrm{AGeV}, 0.2 \mathrm{GeV} / \mathrm{c}$ for $E_{b}=6,30 \mathrm{AGeV}$ and $0.4 \mathrm{GeV} / \mathrm{c}$ for $E_{b}=8 \mathrm{AGeV}$. The $p_{\mathrm{T}}$ and impact parameter (centrality) cuts are chosen to match those used by FOPI, HADES, E895 and STAR 
experiments. The flow signals vary strongly with event generators. At the lowerst energy $E_{b}=$ 1.23 AGeV, which corresponds to that measured by FOPI and HADES at SIS18, the iQMD agrees well with the data. For the SIS100/SIS300 energy range $E_{b}=2-30 \mathrm{AGeV}$ which corresponds to collisions expected for CBM at FAIR, the DCM-QGSM model is the best in describing the E895 and STAR data. This event generator closely follows the experimental data for directed flow $v_{1}$ in contrast to other event enerators and properly describes out-of-plane to in-plane change for the elliptic flow $v_{2}$ data around $E_{b} \sim 4-6 \mathrm{AGeV}$. The availability of fragments in the spectator region and the qualitative agreement with the experimental data for directed flow justifies the use of the DCM-QGSM for the CBM PSD performance study.

\section{PSD performance study}

The PSD performance for the reaction plane reconstruction is studied within CBMROOT environment using GEANT4 Monte-Carlo simulations with the DCM-QGSM event generator. The simulated geometry of the CBM detector includes Au target, dipole magnet, silicon tracking stations (STS) detector, the beam pipe and the PSD.
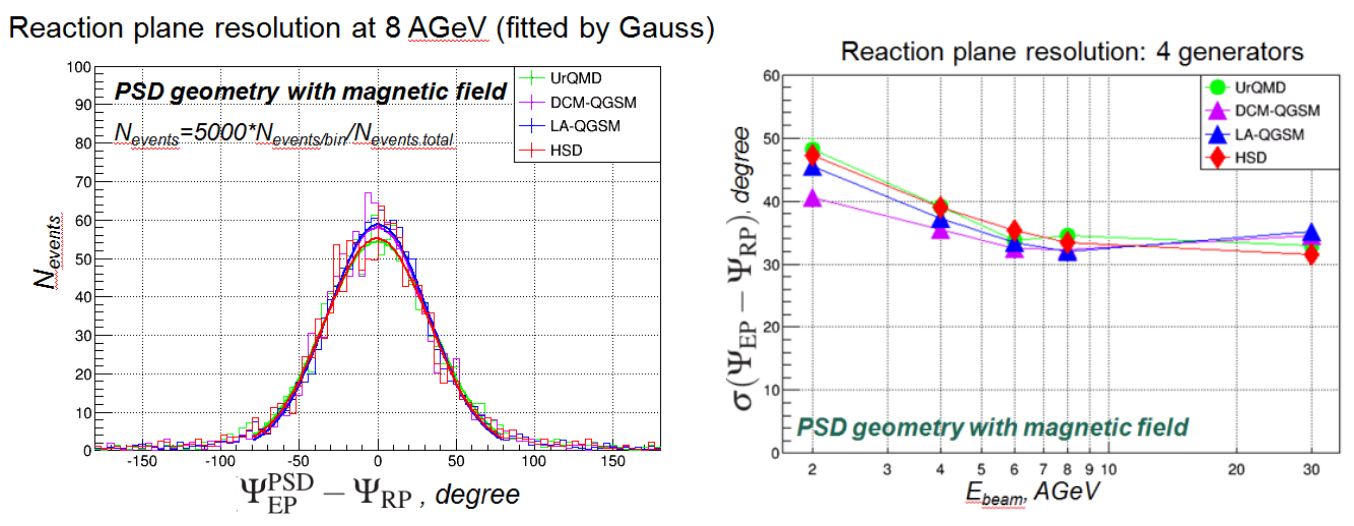

Figure 5: Reaction plane angle determination uncertainty (left) and resolution vs beam energy (right) for various event generators.

Figure 5 shows the reaction plane resolution of the PSD simulated with the above described geometry and enabled magnetic field $B$ using four different event generators. Figure 5(left) shows the distribution for $E_{b}=8 \mathrm{AGeV}$ of the difference $\left(\Psi_{\mathrm{EP}}^{\mathrm{PSD}}-\Psi_{\mathrm{RP}}\right)$ between the reaction plane angle estimate with the PSD $\left(\Psi_{\mathrm{EP}}^{\mathrm{PSD}}\right)$ and the true reaction plane angle $\Psi_{\mathrm{RP}}$ taken from the model input. Figure 5(right) shows the standard deviation $\sigma\left(\Psi_{\mathrm{EP}}-\Psi_{\mathrm{RP}}\right)$ of the gaussian fit to the $\left(\Psi_{\mathrm{EP}}-\Psi_{\mathrm{RP}}\right)$ distribution extracted for different event generators as a function of the beam energy. Reaction plane resolution is below 40 degrees which fulfills the requirements for the flow analysis. Despite the strong variation of the collective flow in models, reaction plane resolution does not differ much between event generators.

\section{Conclusion}

Performance of the PSD for the CBM experiment is studied using different heavy-ion event 
generators and GEANT Monte-Carlo response of the detector components. Collective flow of protons is simulated for semi-central $\mathrm{Au}+\mathrm{Au}$ collisions in the beam energy range $E_{b}=1-30 \mathrm{AGeV}$ using iQMD, UrQMD, DCM-QGSM, LA-QGSM and HSD heavy-ion event generators. The proton flow simulated with various event generators at various energies significantly differs from the experimental data. The DCM-QGSM generator is the most consistent with the experimental data on proton flow in SIS100/SIS300 energy range. Even though directed flow of protons differs significantly for different event generators, the PSD reaction plane resolution for midcentral collisions is simular for different generators and fulfills the requirements for the flow analysis.

\section{Acknowledgments}

We would like to thank for the help in simulating a large ensemble of heavy-ion collisions and helpful discussions: Yvonne Leifels (iQMD), Volker Friese (UrQMD), Marina Golubeva (DCMQGSM), Konstantin Gudima (LA-QGSM), Elena Bratkovskaya (HSD). This work has been supported by the European Community FP7-Capacities, contract HadronPhysics $3 n^{\circ}$ 0283286, grant LG12007 of the Ministry of Education of the Czech Republic and partially by TPU P.L.U.S. program.

\section{References}

[1] C. Pinkenburg et al. [E895 Collaboration], Phys. Rev. Lett. 83, 1295 (1999) [nucl-ex/9903010].

[2] W. Reisdorf et al. [FOPI Collaboration], Nucl. Phys. A 876, 1 (2012) [arXiv:1112.3180 [nucl-ex]].

[3] C. T. Sturm et al. [KAOS Collaboration], Phys. Rev. Lett. 86, 39 (2001) [nucl-ex/0011001].

[4] B. Friman, C. Hohne, J. Knoll, S. Leupold, J. Randrup, R. Rapp and P. Senger, Lect. Notes Phys. 814, pp. 980 (2011).

[5] FAIR, FAIR Baseline Technical Report 2006, www.fair-center.eu/fileadmin/fair/publications_FAIR/FAIR_BTR_6.pdf (2006).

[6] The CBM experiment at FAIR facility, http://www.fair-center.eu/public/experiment-program/nuclear-matter-physics/cbm.html.

[7] F. Guber et.al. [CBM Collaboration], Technical Design Report for the CBM Projectile Spectator Detector (PSD), https://repository.gsi.de/record/109059.

[8] Ch. Hartnack and J. Aichelin, Investigation of the radial expansion of nuclear matter in collisions of heavy nuclei at SIS energies, Rapport Interne SUBATECH 96-08.

[9] M. Bleicher et al., J. Phys. G 25, 1859 (1999) [hep-ph/9909407].

[10] A. V. Dementyev and N. M. Sobolevsky, SHIELD, a Monte Carlo Hadron Transport Code, http://www.inr.troitsk.ru/shield/introd-eng.html.

[11] The HSD model, fias.uni-frankfurt.de/ brat/HSD/index1.html.

[12] A. Sadovsky [HADES Collaboration], Seventeenth Lomonosov Conference On Elementary Particle Physics, Moscow, Russia (2015).

[13] H. Liu et al. [E895 Collaboration], Phys. Rev. Lett. 84, 5488 (2000) [nucl-ex/0005005].

[14] L. Adamczyk et al. [STAR Collaboration], Phys. Rev. Lett. 112, no. 16, 162301 (2014) [arXiv:1401.3043 [nucl-ex]]. 